\title{
ENHANCED ELECTRO-MAGNETIC ENERGY TRANSFER BETWEEN A HOT AND COLD BODY AT CLOSE SPACING DUE TO EVANESCENT FIELDS
}

$$
\text { CONF-981055-- }
$$

J. E. Raynolds

October 1998

DISTRIBUTION OF THIS DOCURENT IS UMLAATED

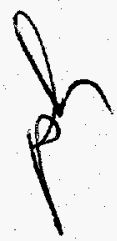

\section{MASTER}

\section{NOTICE}

This report was prepared as an account of work sponsored by the United States Government. Neither the United States, nor the United States Department of Energy, nor any of their employees, nor any of their contractors, subcontractors, or their employees, makes any warranty, express or implied, or assumes any legal liability or responsibility for the accuracy, completeness or usefulness of any information, apparatus, product or process disclosed, or represents that its use would not infringe privately owned rights.

Operated for the U. S. Department of Energy

by KAPL, Inc. a Lockheed Martin company 


\section{DISCLAIMER}

This repor was prepared as an account of work sponsored by an agency of the United States Government. Neither the United States Government nor any agency thereof, nor any of their employees, makes any wartanty, express or implied, or assumes any legal liability or responsibility for the accuracy, completeness, or usefulness of any information, apparatus, product, or process disclosed, or represents that its use would not infringe privately owned rights. Reference berein to any speciric commercial product, process, or service by trade name, trademark. manufac. turer, or otherwise does not necessarily constitute or imply its endorsement, recommendation, or favoring by the United States Governmeat or any agency thereof. The views and opinions of authors expressed herein do not necessarily state or refleet those of the United States Government or any agency thereof. 


\section{DISCLAIMER}

Portions of this document may be illegible in electronic image products. Images are produced from the best available original document. 


\title{
Enhanced Electro-Magnetic Energy Transfer Between a Hot and Cold Body at Close Spacing Due to Evanescent Fields
}

\author{
J.E. Raynolds \\ Lockheed Martin Corp., Schenectady, NY 12301
}

\section{Abstract}

Theoretical studies have demonstrated that the energy transfer between a hot an cold body at close spacing (on the order of the radiation wavelength) can greatly exceed the limit for black body radiation (i.e. Power $=\sigma T^{4}$ ). ${ }^{1-9}$ This effect, due to the coupling of evanescent fields, presents an attractive option for thermo-photovoltaic (TPV) applications (assuming the considerable technical challenges can be overcome). The magnitude of the enhanced energy transfer depends on the optical properties of the hot and cold bodies as characterized by the dielectric functions of the respective materials. The present study considers five different situations as specified by the materials choices for the hot/cold sides: metal/metal, metal/insulator, metal/semiconductor, insulator/insulator, and semiconductor/semiconductor. For each situation, the dielectric functions are specified by typical models. An increase in energy transfer (relative to the black body law) is found for all situations considered, for separations less than one micron, assuming a temperature difference of $1000 \mathrm{C}$. The metal/metal situation has the highest increase vs. separation while the semiconductor/semiconductor has the lowest. Factor-of-ten increases are obtained at roughly 0.1 microns for the metal/metal and roughly 0.02 microns for the metal/semiconductor. These studies are helping to increase the understanding of the close-spaced effect in the context of a radiator/ TPV context.

\section{Enhanced energy transfer at close spacing}

The enhancement at close spacing can be described within the framework of statistical mechanics and classical electromagnetism. Any object at non-zero temperature radiates energy, in the form of fluctuating electromagnetic fields, which are produced by the thermal motion (acceleration) of electric charges within the material. There is a component of the electromagnetic field near the surface of a radiating body which has a fluctuating time dependence, but does not propagate. This so-called "evanescent" field decays exponentially with a characteristic distance on the order of the wavelength. At sufficiently close spacings, the evanescent fields produced in one body interact directly with the electric charges in the other body; and, thus, act as sources of electromagnetic waves. These new waves are transmitted or absorbed depending on the properties of the second body. In a sense, the radiation "tunnels" across the gap and represents a net energy transfer.

\section{Mathematical theory}

Maxwell's equations of electro-magnetism serve as the starting point for the theoretical 
treatment. These are:

$$
\begin{gathered}
\nabla \bullet \dot{D}(\vec{r}, t)=4 \pi \rho(\vec{r}, t), \\
\nabla \bullet \vec{B}(\vec{r}, t)=0, \\
\nabla \times \vec{H}(\vec{r}, t)=\frac{1}{c} \frac{\partial}{\partial t} \vec{D}(\vec{r}, t)+\frac{4 \pi}{c} \vec{\jmath}(\vec{r}, t), \\
\nabla \times \vec{E}(\grave{r}, t)=-\frac{1}{c} \frac{\partial}{\partial t} \vec{B}(\vec{r}, t),
\end{gathered}
$$

where $\vec{E}(†, t), \vec{D}(\xi, t), \vec{H}(\xi, t)$, and $\vec{B}(\xi, t)$, are the electric field, the displacement field, the magnetic field, and the magnetic induction, respectively, at point $\$$ and time $t$ and $c$ is the speed of light. The quantities $\rho(\nexists, t)$, and $\ni(\nexists, t)$ represent the net charge and current densities at point $\rightarrow$ and time $t .10$

By taking the Fourier transform with respect to time of Eqs. 3 and 4 we obtain:

$$
\nabla \times \vec{H}(\vec{r}, \omega)=-i \frac{\omega}{c} \varepsilon(\omega) \vec{E}(\vec{r}, \omega)-i \frac{\omega}{c} \vec{g}(\vec{r}, \omega)
$$

and,

$$
\nabla \times \vec{E}(\xi, \omega)=i\left(\frac{\omega}{c}\right) \vec{H}(\vec{r}, \omega)
$$

where $i=\sqrt{-1}$ and the current density resulting from the fluctuating charges has been taken (for convenience) as $\vec{t}(t, \omega) \equiv-i \omega \vec{g}(t, \omega) / 4 \pi$. Detailed discussions of the fluctuating fields are presented in the book by Rytov. 8

Once the fields have been obtained (through Fourier analysis), the energy transfer (energy per unit area, per unit time) is given by the time average of the Poynting vector,

$$
P=\frac{c}{4 \pi}\langle\vec{E} \times \vec{H}\rangle
$$

where (again) the angled brackets denote time averaging. The Poynting vector contains products of the $g$ fields which give non-vanishing contributions upon time averaging.

The Poynting vector can be evaluated because products of the $g$ fields, when time averaged, are related to the dielectric function. The fluctuation dissipation theorem gives

$$
\left\langle g_{l}\left(\vec{r}_{1}, \omega\right) g_{m}^{*}\left(\vec{t}_{2}, \omega^{\prime}\right)\right\rangle=A(\omega, T) \varepsilon^{\prime \prime}(\omega) \delta_{l m} \delta\left(t_{1}-t_{2}\right) \delta\left(\omega-\omega^{\prime}\right)
$$

where $\varepsilon^{\prime \prime}(\omega)$ is the imaginary part of the dielectric function and $\delta_{l m}$ gives zero unless the two components of the $g$ field are the same $(l=m){ }^{7}$ The Dirac $\delta$-functions $\delta\left(\digamma_{1}-t_{2}\right)$ and $\delta\left(\omega-\omega^{\prime}\right)$, contribute to the integrals in Eq. 7 only when $t_{1}=\nexists_{2}$ and $\omega=\omega^{\prime}$. The frequency and temperature dependent function $A(\omega, T)$ is given by 


$$
A(\omega, T)=2 \hbar \operatorname{coth} \frac{\hbar \omega}{2 k_{B} T}
$$

where $\hbar$ is Planck's constant, $T$ is the temperature, and $k_{B}$ is Boltzmann's constant.

The most general result for the energy transfer between two semi-infinite media with plane parallel surfaces separated by vacuum of thickness $l$, including magnetic effects, is given in Ref. 5 as:

$$
P=\frac{\hbar}{\pi^{2}} \int_{0}^{\infty}\left(\frac{1}{\exp \left(\hbar \omega / k_{B} T_{1}\right)-1}-\frac{1}{\exp \left(\hbar \omega / k_{B} T_{2}\right)-1}\right) M(\omega) \omega d \omega,
$$

where,

$$
\begin{aligned}
M(\omega) & =-\int_{0}^{\infty}\left\{\frac{1}{\left|\Delta_{\varepsilon}\right|^{2}}\left(\frac{p_{1}}{\varepsilon_{1}}-\frac{p_{1}^{*}}{\varepsilon^{*}{ }_{1}}\right)\left(\frac{p_{2}}{\varepsilon_{2}}-\frac{p_{2}^{*}}{\varepsilon^{*}{ }_{2}}\right) .\right. \\
& \left.+\frac{1}{\left|\Delta_{\mu}\right|^{2}}\left(\frac{p_{1}}{\mu_{1}}-\frac{p^{*}{ }_{1}}{\mu^{*}{ }_{1}}\right)\left(\frac{p_{2}}{\mu_{2}}-\frac{p^{*}}{\mu^{*}}\right)\right\}|p|^{2} x d x
\end{aligned}
$$

In Eq. 11, the following definitions hold:

$p=\sqrt{x^{2}-k^{2}}, \quad p_{v}=\sqrt{x^{2}-k^{2} \varepsilon_{v} \mu_{v}}, \quad k=\omega / c, \quad(v=1,2)$,

and,

$$
\Delta_{\varepsilon}=\left(\frac{p_{1}}{\varepsilon_{1}}+p\right)\left(\frac{p_{2}}{\varepsilon_{2}}+p\right) e^{p l}-\left(\frac{p_{1}}{\varepsilon_{1}}-p\right)\left(\frac{p_{2}}{\varepsilon_{2}}-p\right) e^{-p l},
$$

where $l$ is the separation (gap) between the two semi-infinite materials. The expression for $\Delta_{\mu}$ is obtained from $\Delta_{\varepsilon}$ upon making the substitution $\varepsilon_{v} \leftrightarrow \mu_{v}$. For $p_{v}$ the root must be chosen such as to give $\operatorname{Re}\left(p_{v}\right)>0$. In the above equations, the frequency dependence of $\varepsilon_{v}$ and $\mu_{v}$ has been suppressed for brevity. The quantities $T_{1}$ and $T_{2}$ in Eq. 10 are the temperatures of the hot and cold body, respectively. The $k$ defined in Eq. 12 is the wave number of light in vacuum. Thus the quantity $p$ corresponds to propagation in vacuum, while $p_{v}$ corresponds to propagation in medium $v$.

Consider the dependence on the separation $l$ as exhibited in Eq. 13. The variable $p$ for $x<k$, is pure imaginary. Therefore, the exponentials in Eq. 13 are sinusoidal for all values of $l$. This gives rise to a contribution to Eq. 7, which survives at large distances. This contribution is the familiar black body radiation. For $x>k$, the variable $p$ is a positive real number making Eq. 13 an exponentially increasing function of the gap $l$. Therefore, in addition to the black body

Page 3. 
radiation, Eq. 10 contains a contribution which decreases exponentially with separation. There is thus an additional contribution to the energy transfer at close spacing. This enhancement can be significant as is demonstrated in section 6.

\section{Model dielectric functions}

The magnitude of the close-spaced enhancement depends on the properties of the materials used on the hot and cold sides as specified by the frequency dependent dielectric function $\varepsilon(\omega)$. A commonly used model based on classical physics is given by:

$$
\varepsilon(\omega)=1+\frac{4 \pi n e^{2}}{m} \sum_{j}\left(\frac{f_{j}}{\omega_{j}^{2}-i \omega \gamma_{j}-\omega^{2}}\right),
$$

where $n$ is the number of polarizable molecules per unit volume, each containing a fraction $f_{j}$ of electrons having resonant frequency $\omega_{j}$ and damping constant $\gamma_{j}$. The electron mass is $m$ and its charge is $e$.

The dielectric function of Eq. 14 also applies to the special case in which free electrons are present, as in metals. There is no restoring force for free electrons; and, therefore, we must set $\omega_{0} \rightarrow 0$. If in Eq. 14, the contributions due to bound electrons are insignificant in comparison to the free electron term, the following form is obtained for the dielectric function:

$$
\varepsilon(\omega)=1+\frac{4 \pi \sigma \gamma i}{\omega(\gamma-i \omega)}
$$

Equation 15 is the Drude model. ${ }^{11}$ The static conductivity is defined as $\sigma=n f_{0} e^{2} / m \gamma_{0}$. The conductivity $\sigma$ and damping constant $\gamma$ each have units of frequency (the dielectric function is dimensionless).

\section{Evaluation of close-spaced enhanced power transfer for various model materials}

Equation 10 (the energy transfer between a hot and a cold body) was evaluated numerically for various model materials using the analytic forms for the dielectric function described in $t$ section 4. ${ }^{12}$ For convenience, we define dimensionless quantities $l^{\prime}, \omega^{\prime}$, and $P_{3}^{\prime}$ as: $l=l^{\prime} \hbar c / k_{B} T, \omega=\omega^{\prime} k_{B} T / \hbar$, and $P=P^{\prime} P_{b b}$; where $P_{b b}=\pi^{2} k_{B}^{2} T^{4} / 60 \hbar^{3} c^{2}$ is the transfer rate of black body radiation

Equation 15 was used to describe metallic materials. The two quantities $\sigma$ and $\gamma$ were treated as adjustable parameters and were varied to maximize the power transfer (at $l^{\prime}=1.0$ ). Both of these variables have units of frequency and are therefore transformed to dimensionless quantities analogously to $\omega$ (i.e.,substitute $\omega \rightarrow \sigma$, and $\omega \rightarrow \gamma$, respectively in the above definitions of the dimensionless frequency). For a good conductor such as $\mathrm{Cu}$ or $\mathrm{Ag}$ the conductivity and damping constant are given roughly as $\sigma-10^{17} \mathrm{sec}^{-1}$, and $\gamma-10^{13} \mathrm{sec}^{-\mathrm{P}}$, respectively. At $T=1000^{\circ} \mathrm{C}$ the corresponding dimensionless quantities are $\sigma^{\prime}=600$, and $\gamma^{\prime}=0.06$. For the purpose of illustration, Eq. 15 is plotted in Fig. 1 for the arbitrary values $\sigma^{\prime}=1.0$ and $\gamma^{\prime}=2.0$. Figure 1 illustrates the general features of Eq. 15. Both real and imaginary parts tend to unity for high frequencies. The imaginary part is always positive (as is the case for all dielectric functions), 
and diverges at low frequencies, and the real part tends to a constant at zero frequency.

For insulators, a single resonant frequency (and damping constant) was considered in Eq.

14. The dielectric function for an insulator is thus written as

$$
\varepsilon(\omega)=1+\frac{\omega_{p}^{2}}{\left(\omega_{0}^{2}-i \omega \gamma_{0}-\omega^{2}\right)}
$$

where the plasma frequency has been defined as $\omega_{p}=\sqrt{4 \pi n z e^{2} / m}$, and we have taken $f_{0} / z \rightarrow 1$ (all of the valence electrons participating). The dielectric function for an insulator thus has three adjustable parameters: $\omega_{0}, \gamma_{0}$, and $\omega_{p}$. These variables all have dimensions of frequency and are transformed to dimensionless quantities as discussed previously. Typically, the plasma frequency is of the order $10^{16} \mathrm{sec}^{-1}$ which is as large or larger than the frequencies of visible light. The dimensionless plasma frequency is therefore $\omega_{p}^{\prime}=60$. There is a further constraint placed on the resonant frequency and the damping constant which takes the form $\omega_{0}>\gamma_{0} / 2$. This constraint follows from the fact that the response of the system must obey causality (i.e., cause precedes effect).

Another situation of interest is one in which the cold side is a semiconductor. To describe a semiconductor, we start with a published model which gives a good fit to the measured dielectric function for GaAs. ${ }^{13}$ Figure 2 shows the real and imaginary parts of the dielectric function vs. photon energy for $\mathrm{GaAs}$ corresponding to electronic interband transitions (phonon contribution not included).

A model for a smaller band gap material (appropriate to infrared radiation) was obtained by translating the position of the absorption edge and rescaling the energy axis. The semiconductor model dielectric function, so constructed, is plotted in Fig. 3 vs. dimensionless frequency along with the Planck distribution.

\section{Results}

The results obtained by solving Eq. 10 for the energy transfer vs. separation are plotted in Fig. 4 for various combinations of materials on the hot and cold sides. ${ }^{14}$ The hot side temperature was chosen to be $T=1273 \mathrm{~K}\left(1000^{\circ} \mathrm{C}\right)$. A significant enhancement of energy transfer is obtained for all material combinations for separations smaller than one micron. For large separations the energy transfer is somewhat less than that for a black body due to the fact that the materials have emissivity less than unity.

The largest enhancement was found for the situation in which the dielectric function for both the hot and cold side is of the Drude form (Eq. 15). The enhancement (at $l^{\prime}=1.0$ )) was a monotonically increasing function of the damping parameter which reached its maximum asymptotically for roughly $\gamma^{\prime}>50$. The large enhancement (several orders of magnitude) depicted in Fig. 4 corresponds to $\sigma^{\prime}-1.7$ and $\gamma^{\prime} \rightarrow \infty$. These values were obtained by maximizing the energy transfer at $l^{\prime}=1.0$. The dielectric function corresponding to these values is plotted in Fig. 5. These conductivity and damping constant values were used in all subsequent calculations involving metals.

At $l^{\prime}=1.0$ the largest energy transfer (still less than $P_{b b}$ ) was obtained for intermediate values of $\sigma^{\prime}$ (on the order of unity), but was small for large and small values of $\sigma^{\prime}$. This is consistent with the fact that a good metal (large conductivity) has low emissivity. Likewise, in the limit 
$\sigma^{\prime} \rightarrow 0$ we obtain vacuum, and there is thus no radiation.

A large enhanced energy transfer was also found for the situation in which the hot side was a metal characterized by Eq. 15 and the cold side was an insulator characterized by Eq. 16 . The parameters used for the insulator were $\omega_{p}{ }^{\prime}=13.0, \omega_{0}{ }^{\prime}=4.0$, and $\gamma_{0}{ }^{\prime}=1.0$. This dielectric function is plotted in Fig. 6. No attempt was made to find the absolute maximum of the energy transfer for this situation (an optimization in the space of five variables). Indeed, there is no guarantee that the dielectric functions obtained by such a procedure would be physically realizable.

The results for the metal hot side/insulator cold side clearly demonstrate that the enhanced energy transfer is a result of the fact that both materials exhibit absorption (i.e., have dielectric functions with non-zero imaginary parts). It is essentially irrelevant that one is a metal and the other is an insulator. This is consistent with the fact that the source of the energy (as well as the energy transfer) is due to the presence of imaginary part of the dielectric function as indicated by Eq. 8.

The energy transfer for the situations not involving metals is less than that with metals. Undoubtedly this is due to less overlap of the imaginary parts of the dielectric functions. Interestingly, however, the energy transfer with the least enhancement at small separation (i.e., between two semiconductors) is equal to or greater than all the others at the two largest separations considered. This emphasizes the fact that although the source of the energy is the imaginary part of the dielectric function, simple conclusions based on the form of the dielectric function cannot always be drawn. Of course the situation with two semiconductors may not be physically realizable since it assumes that the melting temperature is greater than $T=1000^{\circ} \mathrm{C}$.

\section{Conclusions}

The enhanced energy transfer between a hot and cold body has been evaluated numerically for several model materials for a temperature difference of $1000^{\circ} \mathrm{C}$. The dielectric response of the hot and cold materials dictates the enhancement. Model dielectric functions for a metal, semiconductor, and insulator were considered. The results indicate that significant enhancement occurs only for separations less than one micron.

The largest enhancement occurs for materials which can be described by a metallic (Drude model) dielectric function. The enhancement can be as large as several orders of magnitude (it actually diverges but wasn't proven here ${ }^{5}$ ). The large enhancement occurs also for insulating materials as long as there is absorption (i.e., non-zero imaginary part of the dielectric function). The smallest enhancement was found between two semiconductors. It was argued that this result occurs because this situation exhibits the smallest overlap between the imaginary parts of the dielectric functions. 


\section{Figure captions:}

Figure 1. Real and imaginary parts of model dielectric function (Eq. 15) for dimensionless conductivity and damping constants $\sigma^{\prime}=1.0$, and $\gamma^{\prime}=2.0$ respectively. This plot demonstrates the general features of this model.

Figure 2. Published model dielectric function for GaAs vs. photon energy $(\hbar \omega)$. This model was fit to experimental measurements of the electronic interband contribution to the dielectric function.

Figure 3. Semiconductor model dielectric function (real and imaginary parts) and Planck distribution vs. dimensionless frequency. The semiconductor model was obtained by translating the position of the absorption edge (band gap) and rescaling the frequency variable of the measured dielectric function for GaAs. The Planck distribution gives the intensity of black body radiation vs. frequency. The total power calculated from Eq. 10 is sensitive to the placement of the absorption edge (where the imaginary part of the dielectric function becomes non-zero) relative to the peak of the Planck distribution. The magnitude of the Planck distribution in this figure is arbitrary and was chosen for illustrative purposes.

Figure 4. Total intensity of energy transfer between hot and cold bodies vs. separation for various combinations of materials. The units of separation were obtained by choosing a hot side temperature of $T=1273 \mathrm{~K}\left(1000^{\circ} \mathrm{C}\right)$. The cold side was taken to be at $T=0 \mathrm{~K}$ (although choosing room temperature makes a negligible difference in the result). The intensity is measured in units

of $P_{b b}$ (black body), which for $T=1273 \mathrm{~K}$ has the value $P_{b b}=7.22 \times 10^{-5} \mathrm{~W} / \mathrm{m}^{2}$. A significant enhancement is found for all material combinations for separations smaller than one micron.

Figure 5. Metal (model) dielectric function for the parameters $\sigma^{\prime}=1.7$ and $\gamma^{\prime} \rightarrow \infty$ along with Planck distribution. These parameters were chosen to maximize the energy transfer at $l^{\prime}=1$.

Figure 6. Oscillator model dielectric function (Eq. 14) along with Planck distribution. The parameters are given by $\omega_{p}{ }^{\prime}=13.0, \omega_{0}{ }^{\prime}=4.0$, and $\gamma_{0}{ }^{\prime}=1.0$.

\section{References:}

1. C. E. G. Cravalho, C. L. Tien, R. P. Caren, J. Heat Transfer Trans. ASME, Ser. C 89, 351 
(1967).

2. D. Polder and M. Van Hove, Phys. Rev. B 4, 3303 (1971).

3. R. P. Caren, J. Heat Transfer, Trans. ASME Ser. C 94, 289 (1972).

4. Ibid, p. 295.

5. M. L. Levin, V. G. Polevoi, and S. M. Rytov, Sov. Phys. JETP 52, 1054 (1980).

6. J. J. Loomis and H. J. Maris, Phys. Rev. B 50, 18517 (1994).

7. L. D. Landau, and E. M. Lifshitz, Course of Theoretical Physics, Vol. 5 (Statistical Physics, part 1), (Pergamon, New York, 1988), pp. 384-393.

8. S. M. Rytov, Theory of Electric Fluctuations and Thermal Radiation (Air Force Cambridge Research Center, Bedford, Mass., 1959), AFCRC-TR-59-162.

9. L. D. Landau, and E. M. Lifshitz, Course of Theoretical Physics, Vol. 8 (Electrodynamics of continuous media), (Pergamon, New York, 1960), pp. 367-368.

10. J. D. Jackson, Classical Electrodynamics, Second Edition, (John Wiley \& Sons, New York, 1975), p. 227.

11. N. W. Ashcroft, and N. D. Mermin, Solid State Physics (Saunders College, Philadelphia, 1976), pp. 1-27.

12. Actually, for computational convenience, the equivalent forms given by Eq.'s 21 , and 22 , in Ref. 6 were used.

13. S. Adachi, GaAs and Related Materials: Bulk Semiconducting and Superlattice Properties (World Scientific, Singapore, 1994), pp. 356-366.

14. The energy transfer which is plotted in Fig. 4 represents the total energy transfer. For TPV applications, the more useful quantity is the usable portion of the energy transfer due to absorption above the band gap. 
Metal (model) dielectric function

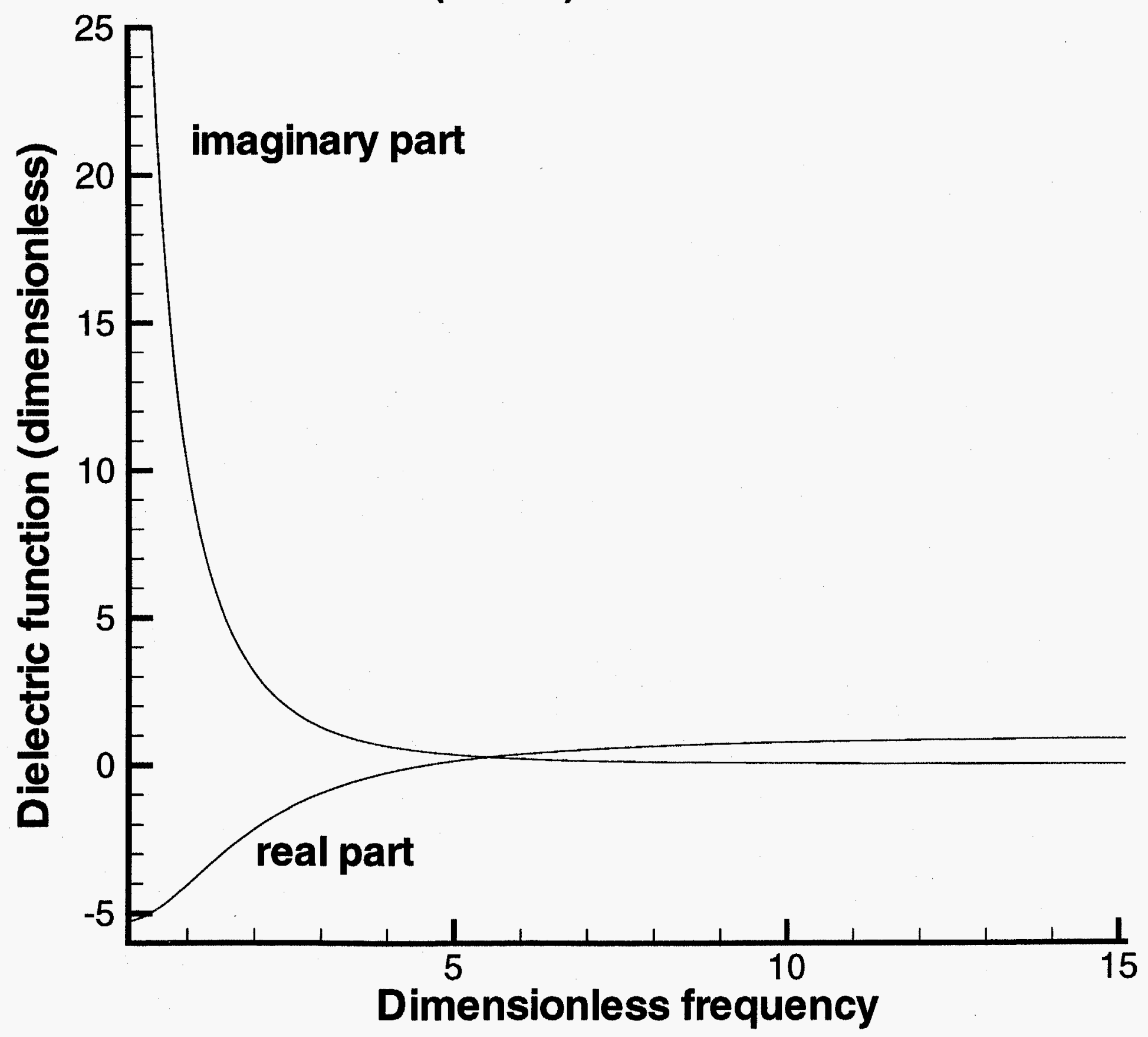




\section{GaAs dielectric function vs. energy}

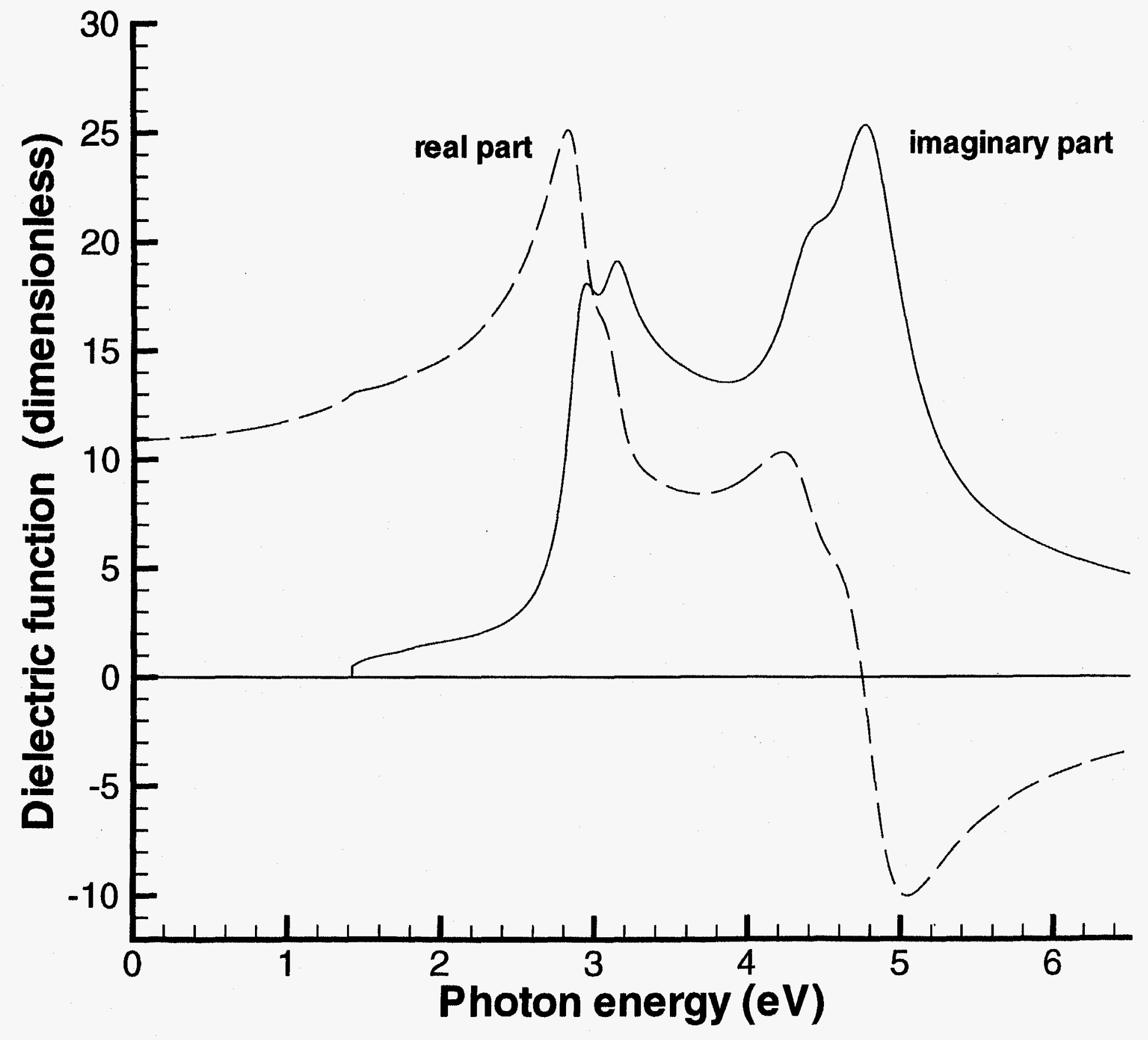


Semiconductor model dielectric function (real and imaginary part) and Planck distribution

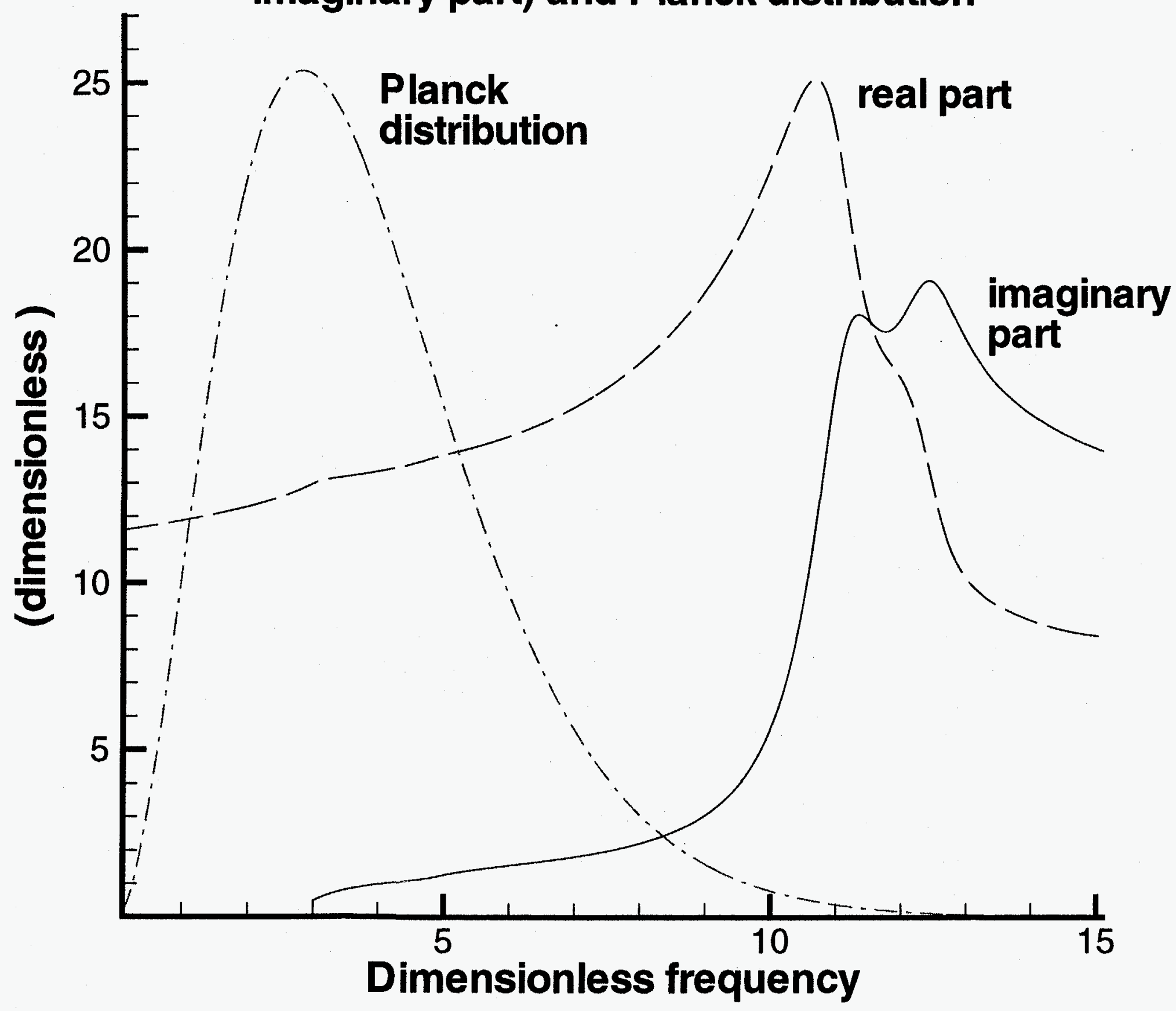




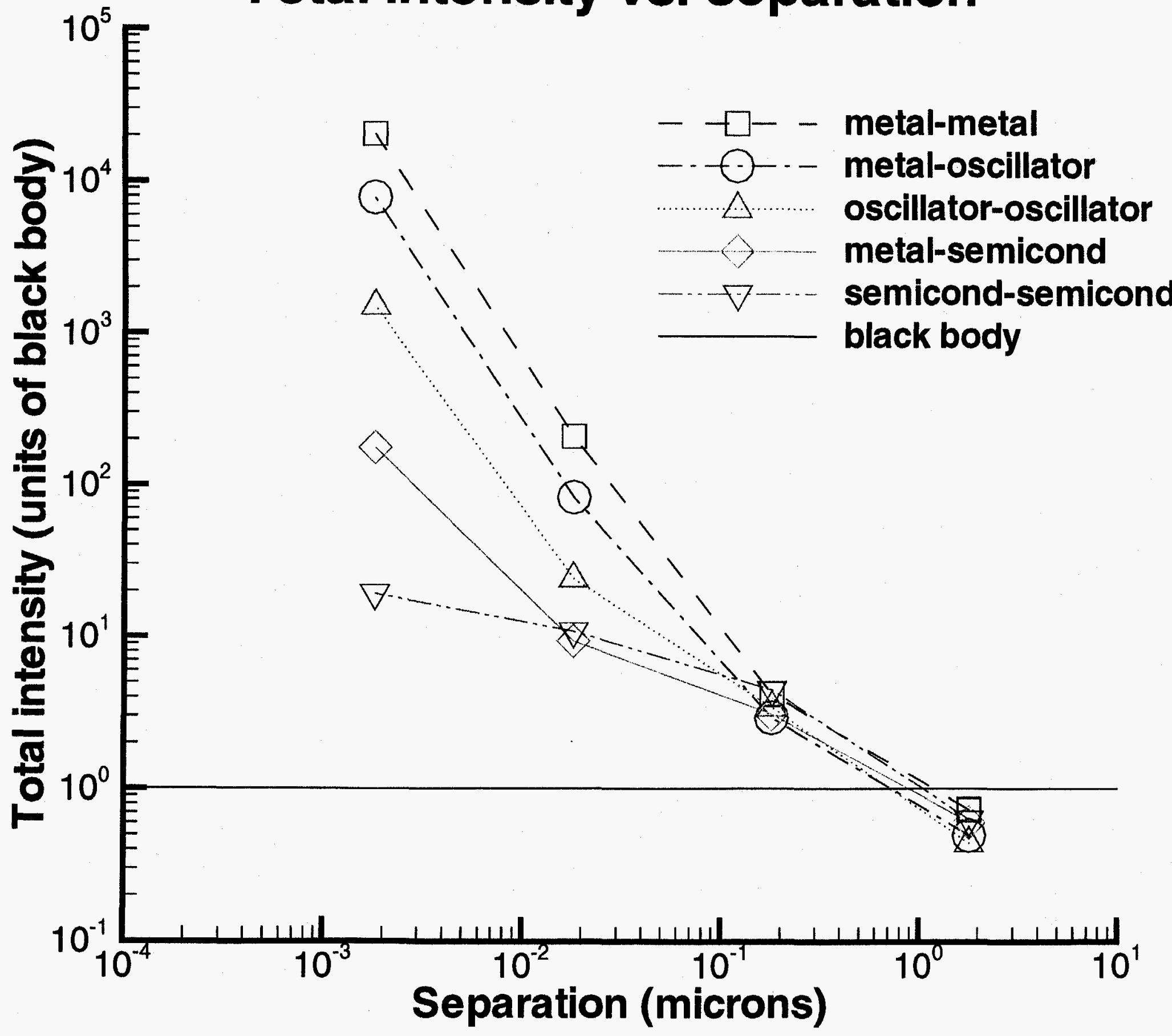

Total intensity vs. separation oscillator-oscillator metal-semicond semicond-semicond black body 


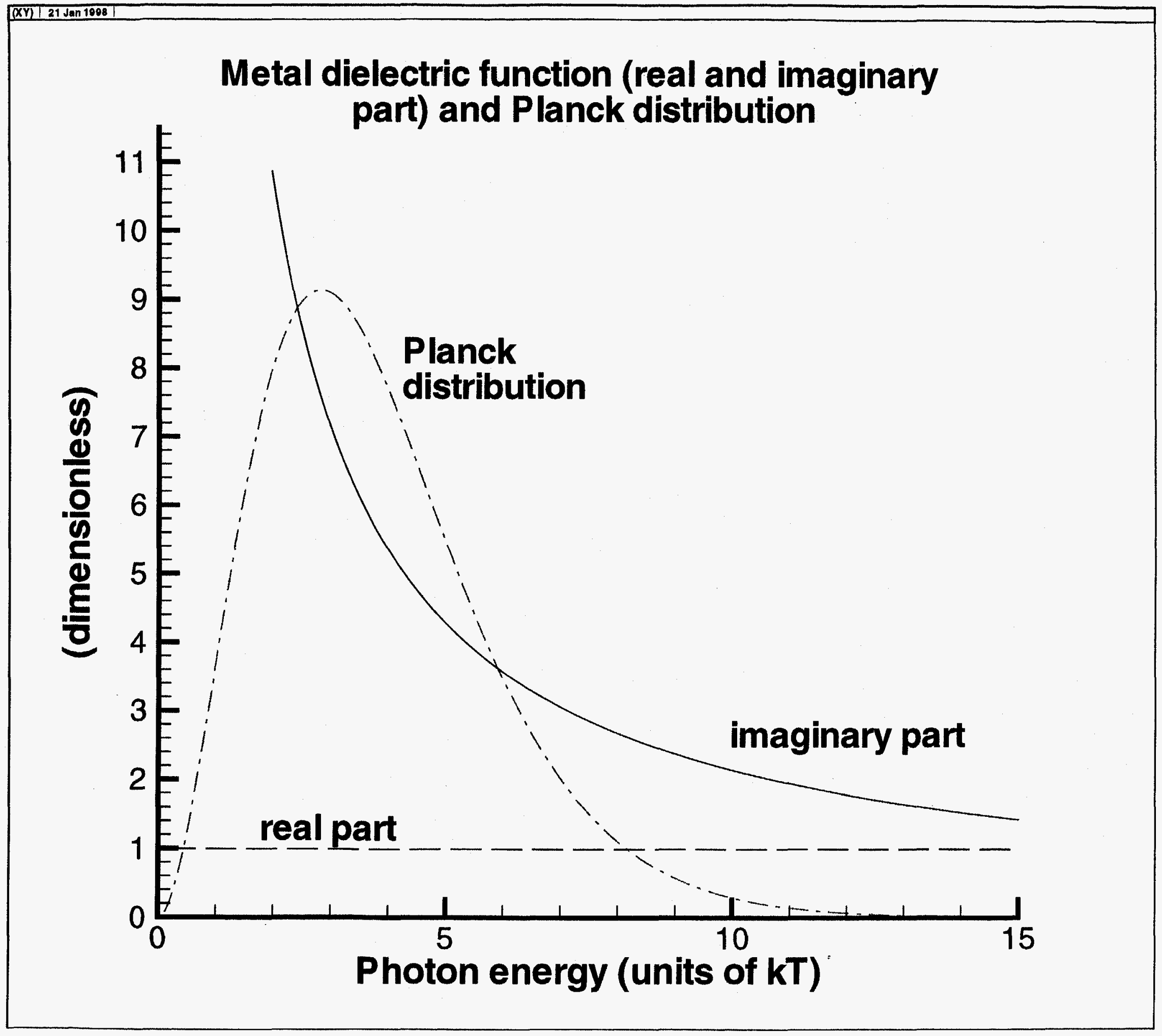




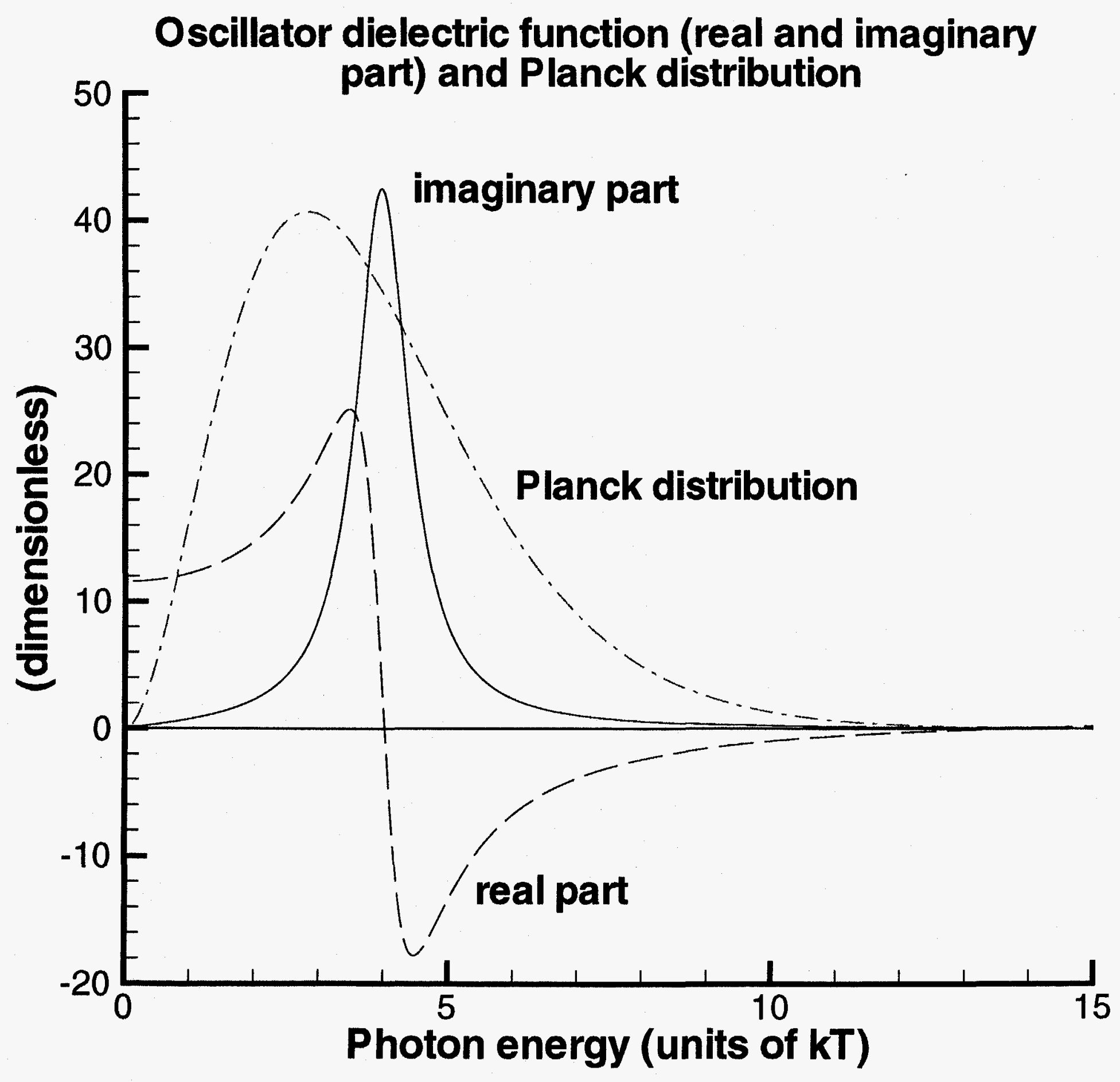

